\title{
Freqüência de relatos de parafunções nos sub- grupos diagnósticos de DTM de acordo com os critérios diagnósticos para pesquisa em disfun- ções temporomandibulares (RDC/TMD)
}

\author{
Raquel Stumpf Branco*, Carla Stumpf Branco**, Ricardo de Souza Tesch***, Abrão Rapoport****
}

\begin{abstract}
Resumo
Introdução: as disfunções temporomandibulares (DTM) podem ser definidas como um conjunto de condições dolorosas e/ou disfuncionais, que envolvem os músculos da mastigação e/ou as articulações temporomandibulares (ATM). Um dos meios usados para o diagnóstico é o "Critério Diagnóstico para Pesquisa em Disfunções Temporomandibulares" (RDC/ TMD). Hábitos parafuncionais são aqueles não relacionados à execução das funções normais do sistema estomatognático. O bruxismo é caracterizado por atividade parafuncional noturna involuntária dos músculos mastigatórios, enquanto o apertamento dentário é considerado uma parafunção diurna envolvendo esta musculatura, embora possa ocorrer também à noite. Objetivos: o objetivo do presente estudo foi avaliar a freqüência do relato de parafunções orais diurna e/ou noturna em pacientes com DTM nos diferentes subgrupos diagnósticos do RDC/TMD. Metodologia: foram utilizados dados provenientes de 217 pacientes que procuraram tratamento na Clínica de DTM e Dor Orofacial da Faculdade de Medicina de Petrópolis, sendo avaliados através do questionário e exame físico que compõem o RDC/ TMD. Resultados: dos 182 pacientes com DTM estudados, 76,9\% relataram algum tipo de parafunção, podendo ser diurna, noturna ou a associação de ambas. A parafunção diurna foi a mais freqüentemente relatada entre os subgrupos de DTM, sendo encontrada em 64,8\% dos casos contra 55,5\% dos casos com relato de bruxismo. O relato de ambas as parafunções foi constatado em 43,4\% dos pacientes com DTM. Conclusões: considerando cada subgrupo diagnóstico, os relatos de parafunções diurna e noturna foram mais freqüentes nos pacientes com dor miofascial.
\end{abstract}

Palavras-chave: DTM. RDC/TMD. Bruxismo. Apertamento dentário.

* Professora convidada do Curso de Especialização em Desordens Temporomandibulares e Dores Orofaciais - Faculdade de Medicina de Petrópolis/RJ. Fisioterapeuta Especializada em Desordens Temporomandibulares e Dores Orofaciais.

** Professora convidada do Curso de Especialização em Desordens Temporomandibulares e Dores Orofaciais - Faculdade de Medicina de Petrópolis/RJ. Fisioterapeuta Especializada em Desordens Temporomandibulares e Dores Orofaciais.

*** Coordenador do Curso de Especialização em Desordens Temporomandibulares e Dores Orofaciais - Faculdade de Medicina de Petrópolis/ RJ. Mestre em Ciências da Saúde - DTM e Dor Orofacial - Hospital Heliópolis/SP.

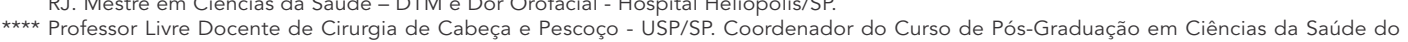
Hospital Heliópolis/SP. 


\section{INTRODUÇÃO E REVISÃO DE LITERATURA}

As disfunções temporomandibulares (DTM) podem ser definidas como um conjunto de condições dolorosas e/ou disfuncionais, que envolvem os músculos da mastigação e/ou as articulações temporomandibulares (ATM) ${ }^{6}$. Estas condições não possuem etiologia ou justificativa biológica comum e, desta forma, caracterizam um grupo heterogêneo de problemas de saúde ${ }^{23}$. Seus sintomas característicos são: dor à palpação muscular e/ou articular, função mandibular limitada e ruídos articulares, com a prevalência total destes sintomas atingindo mais de $75 \%$ da população adulta ${ }^{28}$.

A falta de um perfeito entendimento da relação entre fatores etiológicos e mecanismos fisiopatológicos envolvidos em cada subgrupo de DTM determina que sua classificação atual seja preferivelmente baseada em seus sinais e sintomas do que em sua etiologia". Atualmente, o "Critério Diagnóstico para Pesquisa em Disfunção Temporomandibular" (RDC/TMD) ${ }^{3}$ oferece a melhor classificação para DTM, já que inclui não apenas métodos para a classificação diagnóstica física das DTMs, presentes no seu eixo I, mas ao mesmo tempo métodos para avaliar a intensidade e severidade da dor crônica e os níveis de sintomas depressivos, presentes no seu eixo II.

Denomina-se hábitos parafuncionais aqueles não relacionados à execução das funções normais do sistema estomatognático, como a deglutição, mastigação e fonação. Segundo MacFarlane et al. ${ }^{18}$, fatores mecânicos locais, como os hábitos parafuncionais, apresentam papel importante na etiologia da dor orofacial, podendo sua influência variar segundo a tolerância do paciente à dor $\mathrm{e}$ suas diferentes respostas bioquímicas e fisiológicas a estes fatores ${ }^{5}$. Para Molina et al. ${ }^{22}$, dentre os hábitos orais que afetam as estruturas mastigatórias está o hábito de ranger ou apertar os dentes, também denominado de bruxismo.

De acordo com a Academia Americana de Dor Orofacial e a Associação Americana de Desordens do Sono, o bruxismo é caracterizado por atividade parafuncional noturna e/ou diurna involuntária dos músculos mastigatórios, rítmica ou espasmódica ${ }^{13,22}$, podendo apresentar apertamento e/ou ranger dos dentes ${ }^{2,20,22}$. Hereditariedade, fumo e excesso de consumo de café são alguns fatores de risco para o desenvolvimento do bruxismo ${ }^{13,19,26}$.

O termo bruxismo do sono é preferencialmente utilizado no lugar de bruxismo noturno, pelo fato das pessoas rangerem ou apertarem os dentes quando dormem, de dia ou à noite ${ }^{17}$. É reconhecido como um problema clínico há décadas, embora sua fisiopatologia permaneça controversa ${ }^{13}$. Carlsson et al. ${ }^{1}$ sugeriram que o bruxismo tem regulação central e não periférica. Num episódio de bruxismo, o cérebro é primeiramente ativado, depois é notada uma aceleração cardíaca autonômica e, então, a musculatura mastigatória é fortemente ativada ${ }^{13}$.

Em indivíduos adultos, o sono ocorre em ciclos, em número de 3 a 6, com intervalos de 60 a 90 minutos, incluindo as fases REM (rapid eye movement) e não-REM (estágios 1 e 2 - sono leve; estágios 3 e 4 - sono profundo). Dos episódios de bruxismo, 60 a $80 \%$ ocorrem durante o sono leve, freqüentemente no estágio 2 e durante as trocas de estágios. Podem ocorrer também na fase REM ou no estágio 1 , sendo raramente encontrados nas fases profundas de sono não-REM ${ }^{13}$. A prevalência do bruxismo do sono é de $6 \%$ na população geral ${ }^{17}$. Apresenta diminuição com a idade sendo, segundo alguns estudos, de $28 \%$ em crianças, $14 \%$ em adultos e 3\% acima dos 60 anos de idade ${ }^{13,26}$.

Evidências sugerem que os episódios de bruxismo do sono estão intimamente relacionados ao aumento das atividades eletroencefalográfica, eletromiográfica e freqüência cardíaca, que são parte dos micro-despertares freqüentes durante o sono ${ }^{14}$. Cerca de 60\% dos indivíduos normais apresentam atividade involuntária rítmica da musculatura mastigatória, que ocorre a uma freqüência de 1,8 episódios por hora de sono, a qual pode estar associada à necessidade de estímulo da produção de saliva durante a noite. Em pacientes com bruxis- 
mo do sono esta atividade é 3 vezes mais freqüente, as contrações musculares de maior amplitude e acompanhadas de ruídos dentários ${ }^{15}$.

O bruxismo pode ser avaliado através de questionários de auto-relato, exame clínico oral e estudos desenvolvidos em laboratórios do sono (polissonografias).

A ocorrência de bruxismo do sono baseada no auto-relato pode ser subestimada por ser de difícil diagnóstico em pacientes que dormem sozinhos ou que usam prótese, mas não dormem com esta $^{13}$.

Como descrito por Kato el al. ${ }^{13}$, o Critério Diagnóstico Polissonográfico de Pesquisa para o Bruxismo do Sono investiga a atividade eletromiográfica em músculos mastigatórios (masseter ou temporal) e o tipo de episódio de bruxismo do sono (que pode ser fásico, tônico ou misto). Os registros laboratoriais incluem eletroencefalografia, eletromiografia, eletrocardiograma e sensor térmico, simultâneos com gravações audiovisuais, oferecendo registros altamente controlados para que outras desordens do sono possam ser descartadas e o bruxismo seja discriminado de outras atividades orofaciais que ocorrem durante o sono ${ }^{13}$. Com o estudo polissonográfico, o diagnóstico clínico do bruxismo é corretamente proposto em 83,3\% dos bruxômanos ${ }^{17}$. Entretanto, embora mais confiáveis, têm sua utilidade diagnóstica limitada por sua complexidade de realização e alto custo.

O bruxismo pode também ser clinicamente diagnosticado pela presença de desgaste dentário superior ao considerado normal para a idade, havendo, no entanto, necessidade de diferenciálo do desgaste causado por outros fatores, como dieta específica ou outros hábitos orais ${ }^{13}$. Muitas vezes desgastes dentários evidentes clinicamente não têm correlação com o nível do bruxismo no presente momento, podendo representar registro acumulativo de desgastes parafuncionais e funcionais ${ }^{2}$, pois $40 \%$ da população saudável e que não range os dentes apresentam desgastes dentários ${ }^{13}$.

Em pessoas saudáveis, o contato dentário du- rante o dia é mínimo, somando um tempo total de 17,5 minutos diários, incluindo o tempo de refeições ${ }^{12}$. $\mathrm{O}$ apertamento dentário ou bruxismo diurno acomete indivíduos despertos ${ }^{22}$, podendo ocorrer em circunstâncias diversas e sendo considerado entidade clínica distinta do bruxismo do sono $^{13}$. Sua prevalência é de $20 \%$ na população geral ${ }^{17}$.

Segundo Molina et al..$^{22}$, o apertamento dentário está associado à dor miofascial mastigatória e à artralgia temporomandibular, podendo também ser responsável por dor ou tensão na região cervical. Análises biomecânicas dos componentes da ATM revelaram que estes, normalmente, são capazes de resistir e se adaptar a cargas e pressões funcionais que surgem com movimentos mandibulares de translação. Entretanto, estes tecidos não são capazes de suportar pressões compressivas por um longo período de tempo, como aquelas que podem em alguns indivíduos, em algum grau, estar associadas com apertamento dentário crônico $^{24,29}$.

As pressões intra-articulares maiores que $40 \mathrm{mmHg}$ ultrapassam a pressão dos capilares periféricos e podem causar hipóxia intra-articular temporária, seguida pela re-oxigenação, uma vez que o apertamento é interrompido, com uma resultante liberação de radicais livres ${ }^{8}$. Ao avaliar os niveis de pressão intra-articular presentes nas ATM dos pacientes que estavam acordados e foram submetidos ao procedimento de artrocentese, Nitzan ${ }^{24}$ encontrou que o apertamento voluntário dos dentes produziu altos níveis de pressão intraarticular, maiores que $200 \mathrm{mmHg}$. Existe um dano direto aos componentes do tecido celular e extracelular causados por radicais livres ${ }^{31}$, como o aumento na fricção pela diminuição da lubrificação articular adequada ${ }^{21}$, o que poderia, hipoteticamente, levar ao início do processo de deslocamento anterior do disco articular, como descrito em detalhes por Nitzan ${ }^{25}$.

Citosinas pró-inflamatórias foram isoladas de amostras do líquido sinovial colhido da ATM de 
indivíduos sintomáticos, fato provavelmente relacionado a evidências recentes demonstrando que os radicais livres podem estimular a síntese celular destas proteínas ${ }^{27}$, promovendo um importante efeito pró-inflamatório e de decomposição da estrutura extracelular envolvida no processo de degeneração articular.

O objetivo do presente estudo foi verificar qual a freqüência do relato de parafunções orais diurna e noturna em pacientes com DTM, divididos em diferentes subgrupos diagnósticos, de acordo com o protocolo do RDC/TMD.

\section{MATERIAL E MÉTODOS}

O estudo foi desenvolvido na Clínica de DTM e Dor Orofacial do Ambulatório-Escola da Faculdade de Medicina de Petropólis, em Petrópolis/RJ, e seguiu um protocolo observacional, transversal e descritivo para investigar a freqüência de relatos de parafunção nos diferentes subgrupos diagnósticos, de acordo com o RDC/TMD.

Foi utilizado o banco de dados composto por informações diagnósticas provenientes do questionário e ficha clínica que compõem o RDC/TMD ${ }^{3}$, colhidas entre maio de 2004 e agosto de 2005, provenientes da avaliação de 217 pacientes que

\begin{tabular}{c}
\hline $\begin{array}{c}\text { grupo I } \\
\text { diagnósticos musculares }\end{array}$ \\
\hline la - dor miofascial \\
Ib - dor miofascial com limitação de abertura \\
grupo II \\
deslocamento de disco \\
\hline Ila - deslocamento de disco com redução \\
Ilc - deslocamento de disco sem redução sem limitação de abertura \\
grupo III \\
artralgia, osteoartrite e osteoartrose \\
IIla - artralgia \\
IIlc - osteoartrose da ATM
\end{tabular}

$\overline{\text { Quadro } 1 \text { - Subgrupos diagnósticos de acordo com o RDC/ }}$ TMD (eixo I). procuraram este setor para tratamento. Todos os pacientes assinaram um termo de consentimento livre e esclarecido, permitindo a análise e publicação dos dados colhidos.

Para serem incluídos no presente estudo, os pacientes, de ambos os gêneros, deveriam apresentar idade igual ou superior a 18 anos e ter sido enquadrados em um ou mais dos subgrupos diagnósticos do RDC/TMD.

Foram excluídos os dados daqueles que não tiveram nenhum diagnóstico segundo o RDC/ TMD, num total de 35 pacientes. Permaneceram 182 pacientes selecionados para análise, sendo 166 mulheres e 16 homens, cuja idade variou de 18 a 79 anos, com média de 37 anos.

Ambos os eixos do RDC/TMD foram utilizados para a definição dos subgrupos diagnósticos físicos, descritos no quadro 1, e para a identificação das questões referentes aos relatos de hábitos parafuncionais no questionário, descritos no quadro 2. Não foram feitas distinções, no presente estudo, entre os diagnósticos físicos dentro de cada subgrupo (itens a, b ou c). A análise dos dados e os diagnósticos foram realizados utilizando o software NUS TMD versão 1.1, desenvolvido pela Universidade Nacional de Singapura ${ }^{30}$ para este fim.

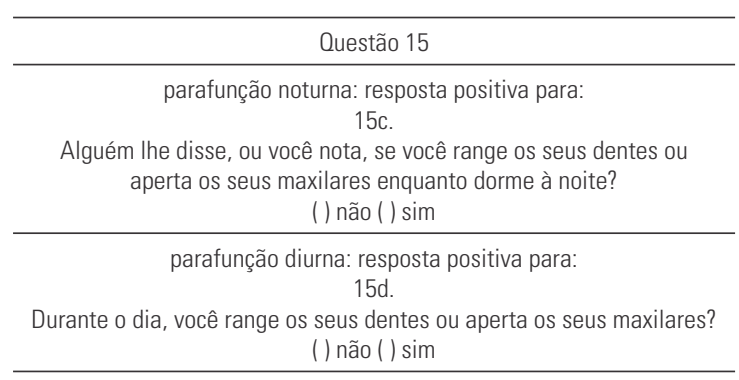

Quadro 2 - Questões referentes aos relatos de parafunções orais, diurna e noturna, de acordo com o RDC/TMD (eixo II). 


\section{RESULTADOS}

A maioria dos pacientes com DTM recebeu diagnóstico associado dos grupos I e III de acordo com o RDC/TMD, ou seja, dor miofascial e alterações álgicas e/ou degenerativas da ATM (Tab. 1), representando 40,7\% da amostra. Em seguida estão os pacientes com dor miofascial isolada, representando $18,7 \%$ do total da amostra. O grupo com menor freqüência de ocorrência, representando apenas 3,3\% da amostra total, foi o que associou o deslocamento do disco e as alterações degenerativas e/ou álgicas da ATM (grupos II e III).

Dos 182 pacientes estudados, 76,9\% $(\mathrm{n}=140)$ relataram algum tipo de parafunção, podendo ser diurna, noturna ou a associação de ambas. A parafunção diurna foi a mais freqüente entre os subgrupos de DTM, sendo encontrada em $64,8 \%$ dos casos contra $55,5 \%$ dos casos com relatos de bruxismo do sono. O relato de ambas as parafunções foi constatado em 43,4\% ( $n=79)$ dos pacientes com DTM.

Considerando a distribuição da freqüência de ocorrência de relato de parafunções, tanto noturna quanto diurna, de acordo com cada subgrupo diagnóstico do RDC/TMD (Tab. 2), foi possível observar no grupo de pacientes com dor miofascial associada às alterações álgicas e/ou degenerativas articulares que $56,8 \%$ dos pacientes relataram ambas as parafunções. Os subgrupos de pacientes com dor miofascial isolada, associação

Tabela 1 - Distribuição da freqüência de ocorrência de diagnósticos de DTM segundo o RDC/TMD.

\begin{tabular}{ccc}
\hline diagnósticos & $\mathbf{n}$ & $\%$ \\
\hline somente grupo I & 34 & 18,7 \\
somente grupo II & 9 & 4,9 \\
somente grupo III & 16 & 8,8 \\
grupos I e II & 18 & 9,9 \\
grupos I e III & 74 & 40,7 \\
grupos II e III & 6 & 3,3 \\
grupos I, II e III & 25 & 13,7 \\
TOTAL & 182 & 100 \\
\hline
\end{tabular}

de dor miofascial e deslocamento do disco articular e associação destas com alterações álgicas e/ou degenerativas articulares, apresentaram também elevada freqüência de relato de ambas as parafunções $(50,0 \%, 33,3 \%$ e 40,0\%, respectivamente).

O grupo que apresentou maior proporção de pacientes sem relato de parafunções $(44,5 \%)$ foi o de deslocamento do disco articular. Entretanto, foi observado que a parafunção apenas noturna foi relatada na maior parte dos casos neste grupo e no grupo composto pela associação deste com dor miofascial (22,2\% para ambos os grupos).

A parafunção diurna mostrou distribuição diferente, sendo encontrada com maior freqüência no grupo de alterações álgicas e/ou degenerativas articulares isoladamente $(56,2 \%)$ e na associação deste com o deslocamento do disco articular (50\%).

O presente estudo demonstrou que 21,3\% $(n=25)$ dos pacientes com dor miofascial isolada ou associada com outro(s) subgrupo(s) de DTM relatam parafunção diurna, $14,5 \%(\mathrm{n}=17)$ relatam parafunção noturna e $64,1 \%(n=75)$ relatam ambas as parafunções, representando $64,2 \%$ ( $\mathrm{n}=$ 117) dos pacientes com estes diagnósticos estudados.

Tabela 2 - Distribuição da freqüência de ocorrência de relatos de parafunções diurna e noturna, nos subgrupos diagnósticos de acordo com o RDC/TMD.

\begin{tabular}{ccccccccc}
\hline & $\begin{array}{c}\text { para- } \\
\text { função } \\
\text { diurna }\end{array}$ & $\begin{array}{c}\text { para- } \\
\text { função } \\
\text { noturna }\end{array}$ & ambas & nenhuma \\
\hline $\begin{array}{c}\text { diagnósticos } \\
\text { somente } \\
\text { grupo I }\end{array}$ & 4 & 11,8 & 3 & 8,8 & 17 & 50,0 & 10 & 29,4 \\
$\begin{array}{c}\text { somente } \\
\text { grupo II }\end{array}$ & 2 & 22,2 & 2 & 22,2 & 1 & 11,1 & 4 & 44,5 \\
somente grupo & 9 & 56,2 & 2 & 12,5 & 2 & 12,5 & 3 & 18,8 \\
III & & & & & & & & \\
grupos I e II & 5 & 27,8 & 4 & 22,2 & 6 & 33,3 & 3 & 16,7 \\
grupos I e III & 10 & 13,5 & 7 & 9,4 & 42 & 56,8 & 15 & 20,3 \\
grupos II e III & 3 & 50,0 & 1 & 16,6 & 1 & 16,6 & 1 & 16,6 \\
grupos I, II e III & 6 & 24,0 & 3 & 12,0 & 10 & 40,0 & 6 & 24,0 \\
TOTAL & 39 & & 22 & & 79 & & 42 & \\
\hline
\end{tabular}

* Porcentagem relativa ao valor total de cada grupo diagnóstico. 
A mesma distribuição em pacientes com alterações álgicas e/ou degenerativas da ATM demonstrou que $29,1 \%$ destes $(n=28)$ relataram parafunção diurna, $13,5 \%(\mathrm{n}=13)$ relataram parafunção noturna, enquanto $57,2 \%(n=55)$ relataram ambas as parafunções, totalizando $52,7 \%(\mathrm{n}=96)$ dos pacientes estudados com este diagnóstico.

No grupo com deslocamento do disco articular, a freqüência de ocorrência de relatos de parafunção foi de $36,3 \%$ ( $n=16)$ para parafunção diurna, $22,7 \%(\mathrm{n}=10)$ para parafunção noturna e $40,9 \%$ $(\mathrm{n}=18)$ para ambas. Assim, apenas $24,1 \%(\mathrm{n}=44)$ dos pacientes com este diagnóstico relataram algum tipo de parafunção.

Dos 173 pacientes incluídos em algum dos subgrupos diagnósticos de DTM dolorosa (dor miofascial, artralgia e osteoartrite), 135 (78,0\%) relatam algum tipo de parafunção oral.

\section{DISCUSSÃO}

Estudos sobre a influência das parafunções na dor orofacial são ainda limitados ${ }^{5}$, porém indicam que o início das DTMs dolorosas possivelmente esteja relacionado ao acúmulo de carga proveniente de hábitos parafuncionais sobre as estruturas do sistema estomatognático ${ }^{22}$. Pesquisas têm sido desenvolvidas no intuito de estabelecer a relação entre parafunções orais, como o bruxismo e o apertamento dentário, e o início ou agravamento de dores orofaciais, mais especificamente da dor muscular mastigatória e artralgia temporomandibular, acompanhada ou não de alterações degenerativas $^{5,22}$.

A polissonografia é citada na literatura ${ }^{13}$ como o método ideal para o diagnóstico do bruxismo do sono. Entretanto, pode também fornecer resultados falso negativos, por se tratar de um problema episódico. Além disso, sua complexidade e alto custo dificultam sua utilização na prática clínica, cabendo ao profissional investigar primeiramente o bruxismo através do auto-relato, conforme demonstrado neste estudo, através do eixo II do RDC/TMD.
Hábitos parafuncionais demonstraram ser freqüentes entre pacientes com diferentes diagnósticos de DTM de acordo com o RDC/TMD. Enquanto a prevalência de bruxismo do sono na população geral é citada na literatura ${ }^{17}$ como estando em torno de $6 \%$, no presente estudo, em pacientes com DTM, a freqüência do relato de bruxismo, que pode ser subestimada, foi de 55,5\%. No que se refere ao apertamento dentário, cuja prevalência na população geral ${ }^{17}$ é de $20 \%$, foi observada também uma elevada freqüência de seu relato $(64,8 \%)$.

No presente estudo, o relato de bruxismo do sono apresentou freqüência de ocorrência inferior $(55,5 \%)$ à apresentada pelo relato de apertamento dentário $(64,8 \%)$. Quando cada diagnóstico do RDC/TMD foi avaliado, associado ou não a outros diagnósticos, a parafunção diurna também foi mais freqüentemente relatada que a do sono. Para Kato et al. ${ }^{13}$, o bruxismo avaliado através do autorelato pode ser subestimado. Segundo Chung et al. ${ }^{2}$, cerca de $70 \%$ dos pacientes com bruxismo do sono não têm conhecimento deste hábito. Como a parafunção diurna acontece com o indivíduo desperto $^{22}$, torna-se mais fácil sua percepção e, conseqüentemente, seu auto-relato é mais freqüente em relação ao bruxismo do sono.

O presente trabalho demonstrou que o relato associado de ambas as parafunções, diurna e do sono, é mais freqüente em todos os subgrupos diagnósticos do que a presença individual de cada uma. Como previamente demonstrado, pacientes com DTM apresentam níveis elevados de atividade nos músculos masseter e temporal em repou$\mathrm{so}^{4,5}$, da mesma forma que pacientes com cefaléia do tipo tensão ${ }^{10}$.

Para Kato et al. ${ }^{13}$, o estalido articular pode ser um sinal indicativo de bruxismo do sono, sugerindo que esta parafunção possa ter alguma relação com o deslocamento do disco articular, tendo o provável mecanismo fisiopatológico determinante desta associação sido previamente descrito ${ }^{25}$. De acordo com pesquisa de Manfredini et al. ${ }^{20}$, 
o bruxismo foi mais freqüentemente encontrado em pacientes com deslocamento do disco articular isolado ou associado a outros grupos. No presente estudo, o bruxismo também foi mais freqüentemente relatado pelo grupo de pacientes com deslocamento do disco articular isolado $(22,2 \%)$ ou associado à dor miofascial $(22,2 \%)$.

Em levantamento epidemiológico realizado por Johansson et al. ${ }^{11}$, através de questionário envolvendo a totalidade da população com 50 anos de idade de dois municípios suecos, foi demonstrado que o bruxismo do sono é um fator de risco significante para artralgia temporomandibular. No presente estudo, a associação das parafunções diurna e noturna foi encontrada com maior freqüência nos pacientes com alterações álgicas e/ou degenerativas articulares, tanto isoladas quanto associadas à dor miofascial. Para Huang et al. ${ }^{9}$, o apertamento dentário está associado tanto à artralgia temporomandibular como à dor miofascial.

$\mathrm{O}$ relato de parafunção diurna foi maior no grupo de pacientes com alterações álgicas e/ou degenerativas da ATM $(56,2 \%)$, confirmando as hipóteses que relacionam o estresse mecânico proveniente do apertamento dentário com o estresse oxidativo articular e o desencadear da seqüência de eventos bioquímicos que irão culminar no processo de degeneração e dor articular ${ }^{24,29}$.

Segundo MacFarlane et al. ${ }^{18}$, as parafunções diurna e do sono foram significativamente associadas à dor orofacial, como à dor articular e à dor durante a mastigação. Setenta e oito por cento dos pacientes com diagnósticos de DTM envolvendo dor orofacial de acordo com o RDC/TMD relataram algum tipo de parafunção, segundo os resultados do presente estudo.

Através deste estudo foi possível demonstrar que a maioria dos pacientes com DTM que procura por tratamento nas clínicas de dor relata algum tipo de parafunção oral, diurna e/ou noturna (76,9\%). Os mecanismos fisiopatológicos responsáveis pela associação de risco destas parafunções com os diferentes subgrupos de DTM ainda não estão bem esclarecidos, mas vários autores consideram o microtrauma relacionado ao bruxismo e o estresse mecânico gerado pelo apertamento dentário como possiveis fatores iniciadores e/ou perpetuadores da dor articular e/ou muscular mastigatória ${ }^{2}$.

Para Kato et al. ${ }^{13}$ e Lavigne et al. ${ }^{16}$, a abordagem de tratamento em longo prazo mais apropriada para o controle de parafunções é a combinação de placas estabilizadoras com estratégias cognitivo-comportamentais (relaxamento, biofeedback, educação) e abordagens farmacológicas, as quais devem ser consideradas de forma global ${ }^{13}$.

As parafunções orais avaliadas no presente estudo podem ser identificadas clinicamente e, portanto, o profissional de saúde deve investigar sinais e sintomas possivelmente relacionados a estas patologias que, uma vez identificadas, devem ser tratadas como fatores de risco para o desenvolvimento das diferentes formas de DTM. Para tal, os protocolos terapêuticos devem ser instituídos com o objetivo de controlar a manifestação destas atividades parafuncionais ou, pelo menos, seus efeitos sobre o sistema estomatognático como forma de prevenção do desenvolvimento de DTM.

A ausência de um grupo controle, composto por indivíduos que não apresentam sinais e sintomas de DTM, foi uma limitação do presente estudo no que se refere à investigação do risco relacionado às parafunções no desenvolvimento das DTMs. Entretanto, a comparação dos resultados obtidos com os valores de referência apresentados na literatura e os resultados obtidos em cada subgrupo diagnóstico de DTM demonstram ser capazes de alcançar os objetivos deste estudo na verificação da distribuição de freqüência de relatos das parafunções e na comprovação de valores elevados destas atividades entre os pacientes com DTM.

\section{CONCLUSÕES}

Segundo os resultados do presente estudo, a parafunção diurna foi mais freqüentemente relatada no subgrupo de pacientes com diagnóstico 
de alterações álgicas e/ou degenerativas da ATM, enquanto a parafunção noturna foi mais freqüentemente relatada nos subgrupos de pacientes com deslocamento do disco articular isoladamente ou em associação com a dor miofascial.

Em relação às distintas possibilidades de associações de diagnósticos de DTM de acordo com o $\mathrm{RDC} / \mathrm{TMD}$, o grupo que relatou maior freqüência de ambos os hábitos parafuncionais foi o de pa- cientes com dor miofascial associada às alterações álgicas e/ou degenerativas da ATM. Considerando cada subgrupo diagnóstico isoladamente, os relatos de parafunções diurna e noturna foram mais freqüentes nos pacientes com dor miofascial.

\title{
Frequency of relates of parafunctions in the diagnostic subgroups of TMD accord- ing to Research Diagnostic Criteria for Temporomandibular Disorders (RDC/TMD)
}

\begin{abstract}
Introduction: temporomandibular disorders (TMD) can be defined as a group of painful and/or dysfunctional conditions that involve masticatory muscles and/or the temporomandibular joints (TMJ). One of the methods used to the diagnostic is the "Research Diagnostic Criteria for Temporomandibular Disorders" (RDC/TMD). Parafunctional habits are those not related to the execution of normal functions of stomatognatic system. Bruxism is characterized by nocturnal involuntary parafunctional activity of masticatory muscles, while clenching is considered as a diurnal parafunction involving this musculature, although this may also occur at night. Objective: the goal of the present study was to evaluate the frequency of relates of diurnal and/or nocturnal oral parafunctions in patients with TMD in different diagnostic subgroups of RDC/TMD. Methodology: it has been used data from 217 patients that seek for treatment at TMD and Orofacial Pain Clinic of Petropolis Medicine School, being evaluated through questionnaire and physical examination that compose RDC/TMD. Results: from 182 TMD patients studied, 76.9\% has related some kind of parafunction, that could be diurnal, nocturnal or both. Diurnal parafunction was the most frequent related among TMD subgroups, present in $64.8 \%$ of cases against $55.5 \%$ of cases with relates of bruxism. Relate of both parafunctions was verified in $43.4 \%$ of TMD patients. Conclusion: regarding each diagnostic subgroup, relates of diurnal and nocturnal parafunctions has been more frequent in patients of miofascial pain group.
\end{abstract}

Key words: TMD. RDC/TMD. Bruxism. Clenching.

\section{REFERÊNCIAS}

1. CARLSSON, G. E.; MAGNUSSON, T.; EGERMARK, I. Prediction of demand for treatment of temporomandibular disorders base on a 20-year follow-up study. J. Oral Rehabil., Oxford, v. 31 p. 511-517, 2004

2. CHUNG, S. C.; KIM, Y. K.; KIM, H. S. Prevalence and patterns of nocturnal bruxofacets on stabilization splints in temporomandibular disorder patients. Cranio, Chattanooga, v. 18, no. 18, no. 2, p. 92-97, 2000

3. DWORKIN, S. F.; LERESCHE, L. Research diagnostic criteria for temporomandibular disorders: review, criteria, examinations and specifications critique. J. Craniomandib. Disord. Facial Oral Pain, Lombard, v. 6, no. 4, p. 300-355, 1992.

4. GLAROS, A. G.; BAHARLOO, L.; GLASS, E. G. Effect of parafunctional clenching and estrogen on temporomandibular disorder pain. Cranio, Chattanooga, v. 16, no. 2, p. 78-83, 1998.
5. GLAROS, A. G.; TABACCHI, K. N.; GLASS, E. G. Effect of parafunctional clenching on TMD. J. Orofac. Pain, Carol Stream, v. 12 , no. 2 , p. $145-152,1998$.

6. GOLDSTEIN, B. H. Temporomandibular disorders: a review of current understanding. Oral Surg. Oral Med. Oral Pathol. Oral Radiol. Endod., St. Louis, v. 88, p. 379-385, 1999.

7. GREENE, C. S. The etiology of temporomandibular disorders: implications for treatment. J. Orofac. Pain, Carol Stream, v. 5 no. 2, p. 93-105, 2001.

8. HALLIWELL, B.; GUTTERIDGE, J. M. The definition and measurement of antioxidants in biological systems. Free Radic. Biol. Med., Tarrytown, v. 18, no. 1, p. 125-126, 1995.

9. HUANG, G. J.; LE RESCHE, L.; CRITCHLOW, C. W.; MARTIN, M. D.; DRANGSHOLT, N. T.Risk factors for diagnostic sugroups of painful temporomandibular disorders (TMD). J. Dent. Res. 
Alexandria, v. 81, no. 4, p. 284-288, 2002

10. JENSEN, R. Pathophysiological mechanisms of tension-type headache: a review of epidemiological and experimental studies. Cephalalgia, Oslo, v. 19, no. 6, p. 602-621, 1999

11. JOHANSSON, A.: UNELL, L.; CARLSSON, G. E.; SODERFELDT, B.; HALLING, A. Gender difference in symptoms related to temporomandibular disorders in a population of 50-year-old subjects. J. Orofac. Pain, Carol Stream, v. 17, no. 1, p. $29-35,2003$

12. KATO, T.; THIE, N. M.; HUYNH, N.; MIYAWAKI, S.; LAVIGNE, G. J. Topical review: sleep bruxism and the role of peripheral sensory influences. J. Orofac. Pain, Carol Stream, v. 17, no. 3, p. 191-213, 2003

13. KATO, T.; THIE, N. M. R.; MONTPLAISIR, J. Y.; LAVIGNE, G. J. Bruxism and orofacial movements during sleep. Dent. Clin. North Am., Philadelphia, v. 45, no. 4, p. 657-684, 2001.

14. LAVIGNE, G.; ZUCCONI, M.; CASTRONOVO, C.; MANZINI, C.; MARCHETTINI, P.; SMIRNE, S. Sleep arousal response to experimental thermal stimulation during sleep in human subjects free of pain and sleep problems. Pain, Amsterdam, v. 84 , no. $2 / 3$, p. $283-290,2000$.

15. LAVIGNE, G. J.; GUITARD, F.; ROMPRÉ, P. H. Validity in sleep bruxism activity over time. J. Sleep Res., Oxford, v. 10, p. 237-244, 2001.

16. LAVIGNE, G. J.; KATO, T.; KOLTA, A.; SESSLE, B. J. Neurobiological mechanisms involved in sleep bruxism. Crit. Rev. Oral Biol. Med., Alexandria, v. 14, no. 1, p. 30-46, 2003

17. LAVIGNE, G. J.; ROMPRE, P. H.; MONTPLAISIR, J. Y. Sleep bruxism: validity of clinical research diagnostic criteria in a controlled polysomnographic study. J. Dent. Res., Alexandria, v. 75, no. 1, p. 546-552, 1996.

18. MACFARLANE, T. V.; BLINKHORN, A. S.; DAVIES, R. M.; WORTHINGTON, H. V. Association between local mechanical factors and orofacial pain: survey in the community. J. Dent. Bristol, v. 31, no. 8, p. 535-542, 2003.

19. MAJOR, M.; ROMPRE, P. H.; GUITARD, F.; TENBOKUM, L.; O'CONNOR, K.; NIELSEN, T.; LAVIGNE, G. J. A controlled daytime challenge of motor performance and vigilance in sleep bruxers. J. Dent. Res., Alexandria, v. 78, no. 11, p. 1754-1762, 1999

20. MANFREDINI, D.; CANTINI, E.; ROMAGNOLI, M.; BOSCO, $M$. Prevalence of bruxism in patients with different research diagnostic criteria for temporomandibular disorders (RDC)
TMD) diagnoses. Cranio, Chattanooga, v. 21, no. 4, p. $279-285,2003$

21. MILAM, S. B.; ZARDENETA, G.; SCHMITZ, J. P. Oxidative stress and degenerative temporomandibular joint disease: a proposed hypothesis. J. Oral Maxillofac. Surg., Philadelphia, v. 56, p. $214-223,1998$.

22. MOLINA, O. M.; SANTOS, J.; MAZZETTO, M.; NELSON, S.; NOWLIN, T.; MAINIERI, E. T. Oral jaw behaviors in TMD and bruxism: a comparison study by severity of bruxism. J. Craniomandibular Pract., Chattanooga, v. 19, no. 2, p. 114-122, 2001.

23. NATIONAL INSTITUTE OF HEALTH. Technol assess statement. management of temporomandibular disorders. [S. I.], 1996

24. NITZAN, D. W. Intra-articular pressure in the functioning human temporomandibular joint and its alteration by uniform elevation of the occlusal plane. J. Oral Maxillofac. Surg., Philadelphia, v. 52, p. 671-679, 1994.

25. NITZAN, D. W. The process of lubrication impairment and its involvement in temporomandibular joint disc displacement: a theoretical concept . J. Oral Maxillofac. Surg., Philadelphia, v. 59, no. 1, p. 36-45, 2001.

26. OHAYON, M. M. Risk factors for sleep bruxism in the general population. Chest, Northbrook, v. 119, no. 1, p. 53-61, 2001.

27. REMACLE, J.: RAES, M.: TOUSSAINT, O.: RENARD, P.: RAO, G. Low levels of reactive oxygen species as modulators of cell function. Mutat. Res., Amsterdam, v. 316, no. 3, p. 103-122, 1995.

28. SOLBERG, W. K. WOO, M. W.; HOUSTON, J. B. Prevalence of mandibular dysfunction in young adults. J. Am. Dent. Assoc. Chicago, v. 98, no. 1, p. 25-34, 1979.

29. STEGENGA, B.; DE BONT, L. G.; BOERING, G.; VAN WILLIGEN, J. D. Tissue responses to degenerative changes in the temporomandibular joint: a review. J. Oral Maxillofac. Surg. Philadelphia, v. 49, no. 10, p. 1079-1088, 1991

30. YAP, A.U.; TAN, K. B.; HOE, J. K.; YAP, R. H.; JAFFAR, J. On-line computerized diagnosis of pain-related disability and psychological status of TMD patients: a pilot study. J. Oral Rehabil., Oxford, v. 28, p. 78-87, 2001.

31. ZARDENETA, G.; MUKAI, H.; MARKER, V.; MILAM, S. B. Protein interactions with particulate Teflon: implications for the foreign body response. J. Oral Maxillofac. Surg., Philadelphia, v. 54, no. 7, p. 873-878, 1996.

\section{Endereço para correspondência}

Raquel Stumpf Branco

Av. Portugal, 390 - Valparaíso

CEP: 25.655-374 - Petrópolis / RJ

E-mail: stumpfbranco@yahoo.com.br 